\title{
Prevención de embolia pulmonary trombosis venosa profunda con aspirina en baja dosis: Pulmonary Embolism Prevention (PEP) trial
}

Prevention of pulmonary embolism and deep venous thrombosis with low dose aspirin: Pulmonary Embolism Prevention (PEP) trial. The Lancet 2000; 355: 1295-1302.:

\section{Objetivo}

Evaluar la eficacia del tratamiento preventivo con aspirina en baja dosis para reducir el riesgo de embolia pulmonar y trombosis venosa profunda en pacientes con fractura y prótesis de cadera.

\section{Diseño}

Ensayo clínico randomizado placebo-control doble ciego.

\section{Lugar}

Estudio multicéntrico realizado en 148 hospitales de Australia, Nueva Zelandia, Sudáfrica, Suecia y Inglaterra.

\section{Pacientes}

Se incluyeron 13356 pacientes con fractura de cadera y 4088 pacientes con artroplastias de cadera electivas después de la internación o antes de la cirugía programada, previa aceptación del consentimiento informado, para cumplir 5 semanas del tratamiento. Se aceptó incluir pacientes que recibían otros tratamientos de profilaxis para enfermedad tromboembólica venosa (ETV) o que estaban usando aspirina o anti-inflamatorios no esteroideos.

\section{Intervención}

Se randomizó telefónicamente a los pacientes para recibir aspirina recubierta $160 \mathrm{mg} /$ día o placebo, el tratamiento se iniciaba preoperatoriamente continuando hasta cumplir 35 días, a excepción de muerte previa o desarrollo de indicaciones o contraindicaciones para recibir aspirina.

\section{Medición de resultados principales}

El objetivo principal fue evaluar el desarrollo de morbilidad y mortalidad hasta el día 35. El seguimiento de eventos no fatales como trombosis venosa profunda (TVP), embolia pulmonar (TEP), infarto de miocardio, accidente cerebrovascular y complicaciones hemorrágicas se realizó solamente durante la estadía hospitalaria, a diferencia de la mortalidad cuya evaluación se continuó durante los 35 días.

\section{Principales resultados}

En los pacientes con fractura de cadera que recibieron prevención con aspirina se observó una reducción de TEP del 43\% (IC95\% $18-60, p=0.002)$ y de TVP sintomática del $29 \%$ (IC95\% $3-48$, $p=0.03)$. Se confirmo TEP o TVP en $105 / 6.679$ pacientes $(1,6 \%)$ que recibieron aspirina y en 165/6.677 (2,5\%) que recibieron placebo, lo cual representa una reducción absoluta de 9 por cada 1000 casos y una reducción proporcional del 36\% (IC95\% 19-50, $\mathrm{p}=0.0003$ ). Similar proporción del efecto se vio en la mayoría de los subgrupos, incluyendo a los que recibieron heparina subcutánea. La aspirina previno 4 TEP fatales por cada 1000 pacientes (18 muertes en el grupo con aspirina vs 43 en el grupo placebo) lo cual representa una reducción del 58\% (IC95\% 27-76, p=0.002) sin efecto aparente sobre muertes por otra enfermedad vascular RR 1.04 (IC95\% 0.86-1.26) y causa no vascular RR 1.01 (0.841.23). Las muertes por sangrado fueron pocas (13 en grupo con aspirina vs 15 en el grupo placebo), pero hubo un exceso de 6 episodios postoperatorios de sangrado transfundidos por cada 1000 pacientes tratados a aspirina $(p=0.04)$. En los pacientes con artroplastia la frecuencia de tromboembolismo venoso fue baja, pero el efecto proporcional de la aspirina fue comparable al de los pacientes con fractura de cadera.

\section{Conclusiones}

El tratamiento preventivo en pacientes con cirugía por fractura de cadera y artroplastia con aspirina reduce en al menos un tercio el riesgo de TEP y TVP durante el período donde este riesgo esta incrementado.

Fuente de financiamiento: no reportada

\section{Comentario}

El estudio demuestra la reducción del riesgo de TEP (incluyendo eventos fatales) y TVP sintomática con baja dosis de aspirina, confirmando el resultado de meta-analisis previos 1,2 . Este beneficio según el autor, ocurrió en los diferentes subgrupos incluyendo aquellos que habían recibido otras medidas de tromboprofilaxis, como también se puso de manifiesto en meta-análisis previos con pacientes sometidos a cirugía ortopédica post-traumatica y electiva, cirugía general y pacientes con condiciones médicas de alto riesgo. Sin embargo, el beneficio en el subgrupo tratado con heparina no fue igual, ya que la aspirina solo mostró beneficio cuando se administró tratamiento con heparina no fraccionada (HNF) no observándose beneficio cuando se uso con heparinas de bajo peso molecular (HBPM). Teniendo en cuenta que las HBPM han demostrado tener una mayor o similar eficacia que la HNF en la prevención de TVP/TEP en este tipo de procedimientos, se debería estudiar si es útil la administración de aspirina en este subgrupo de pacientes $3,4,5,6,7,8$. Por su diseño, este trabajo no tiene poder para medir el impacto en la reducción de la mortalidad secundaria a eventos vascülares (IAM, accidente cerebrovascular) con el uso de bajas dosis de aspirina y por esta razón podría explicarse esa falta de efecto en la prevención de eventos vasculares. Si bien con la administración de aspirina en las 48 horas preoperatorias se obtuvo una reducción más significativa de ETV que su inicio en el post-quirúrgico, también se observó un incremento del requerimiento transfusional secundario al sangrado en el grupo con aspirina, sin que esto significara un aumento de la mortalidad. Otro hecho a destacar, es que el beneficio de su administración se vio después de la primer semana de tratamiento, período donde por lo general se suelen discontinuar las otras medidas de profilaxis de ETV. Estos resultados proveen una buena evidencia sobre la utilidad de la aspirina en la profilaxis de ETV, sin embargo su uso y rol aun no está definido, ya que es necesario documentar su eficacia para patologías específicas en forma comparativa a las otras medidas profilácticas como por ejemplo el uso de HBPM. Esto permitirá conocer con más precisión el grado de efectividad y contribuirá a aclarar cual será su futuro en la profilaxis de la ETV.

\section{Dr. Jorge Alberbide [ Servicio de Hematología. Hospital Italiano de Buenos Aires ]}

\section{Referencias}

1. Powers PJ, Gent M, Jay RM, Julian DH, Turpie AG, Levine M. Hirsh J.A randomized trial of less intense postoperative warfarin or aspirin therapy in the prevention of venous thromboembolism after surgery for fractured hip. Arch Intern Med 1989; 149: 771-4.

boembolism after surgery for fractured hip. Arch Intern Med 1989; 149: 771-4.
2. MacMahon S, Rodgers A, Collins R, Farrell B.Antiplatelet therapy to prevent thrombosis after hip fracture. Rationale for a randomised trial. J Bone Joint Surg Br 1994; 76 : $521-4$.

2. MacMahon S, Rodgers A, Collins R, Farrell B.Antiplatelet therapy to prevent thrombosis after hip fracture. Rationale for a randomised trial. J Bone Joint Surg Br 1994; $76: 521-4$.
3. Levine MN, Hirsh J, Gent M, et al. Prevention of deep vein thrombosis after elective hip surgery: a randomized trial comparing low molecular weight heparin with standard unfractionated heparin. Ann Intern Med 1991; 114: 545-551

4. Planes A, Vochelle N, Mazas F, et al. Prevention of postoperative venous thrombosis: a randomized trial comparing unfractionated heparin with low molecular weight heparin in patients undergoing total hip replacement. Thromb Haemost 1988; 60: 407-410.

5. Eriksson BI, Kalebo P, Anthmyr BA, et al. Prevention of deep vein thrombosis and pulmonary embolism after total hip replacement: comparison of low-molecular-weight heparin and unfractionated heparin. J Bone Joint Surg Am 1991; 73: 484-493.

6. Leyvraz PF, Bachmann F, Hoek J, et al. Prevention of deep vein thrombosis after hip replacement: randomized comparison between unfractionated heparin and low molecular weight heparin. BMJ 1991; 303: 543-548.

7. Dechavanne M, Ville D, Berruyer M, et al. Randomized trial of low molecular weight heparin (Kabi 2165) versus adjusted dose subcutaneous standard heparin in the prophylaxis of deep vein thrombosis after elective hip surgery. Haemostasis 1989;1:5-12. 